\title{
CHEMNANOMAT
}

CHEMISTRY OF NANOMATERIALS FOR ENERGY, BIOLOGY AND MORE

www.chemnanomat.org

\section{Accepted Article}

Title: Self-assembly of clicked star-shaped triazines into functional nanostructures

Authors: Martín Castillo-Vallés, Eduardo Beltrán, Jesús Cerdá, Juan Aragó, Pilar Romero, José Luis Serrano, Enrique Ortí, Raquel Giménez, and Teresa Sierra

This manuscript has been accepted after peer review and appears as an Accepted Article online prior to editing, proofing, and formal publication of the final Version of Record (VoR). This work is currently citable by using the Digital Object Identifier (DOI) given below. The VoR will be published online in Early View as soon as possible and may be different to this Accepted Article as a result of editing. Readers should obtain the VoR from the journal website shown below when it is published to ensure accuracy of information. The authors are responsible for the content of this Accepted Article.

To be cited as: ChemNanoMat 10.1002/cnma.201800484

Link to VoR: http://dx.doi.org/10.1002/cnma.201800484 


\section{WILEY-VCH}

DOI: 10.1002/ ((please add manuscript number))

Article type: Full Paper

\section{Self-assembly of clicked star-shaped triazines into functional nanostructures}

Martín Castillo-Vallés, Eduardo Beltrán, Jesús Cerdá, Juan Aragó, Pilar Romero, José Luis Serrano, Enrique Orti* Raquel Giménez* and Teresa Sierra*

M. Castillo-Vallés, Dr. E. Beltrán, Dr. P. Romero, Prof. J. L. Serrano, Dr. R. Giménez and Dr. T. Sierra

Departamento de Química Orgánica, Instituto de Ciencia de Materiales de Aragón (ICMA) Universidad de Zaragoza - CSIC

50009 Zaragoza, Spain

E-mail: rgimenez@unizar.es, tsierra@unizar.es

Dr. E. Beltrán

Present address: Merck Chemicals Ltd., University Parkway, Southampton SO16 7QD, UK

J. Cerdá, Dr. J. Aragó, Prof. E. Ortí

Instituto de Ciencia Molecular

Universidad de Valencia

E-mail: enrique.orti@uv.es

Keywords: self-assembly, triazine, nanoparticle, nanotube, AIE

Abstract: Two non-amphiphilic star-shaped 2,4,6-tris(1,2,3-triazol-4-yl)-1,3,5-triazines

showing different behavior in terms of self-assembly and luminescent properties are described.

They aggregate in the liquid phase to form low-dimensional nanostructures with a variety of morphologies, such as spherical particles, one-hole hollow spheres, toroids, twisted fibers or helical nanotubes, just by varying the conditions of a straightforward reprecipitation method. Aggregation has an opposite effect concerning the fluorescent properties of the proposed compounds, either causing the enhancement or the quenching of the emission after the selfassembly. Quantum chemical calculations have been also performed to assist in the structural and electronic characterization of the two star-shaped compounds. 


\section{WILEY-VCH}

\section{Introduction}

In recent years, nanomaterials have aroused great interest due to their unique properties in comparison with their macroscopic counterparts. Organic nanomaterials hold some advantages such as a great variety of chemical structures, low processing costs and tuneable electronic and optical properties, that make them interesting for technological applications. ${ }^{[1-4]}$ Indeed, nanoaggregates made of $\pi$-conjugated organic molecules are being investigated as non-linear optical materials, ${ }^{[5,6]}$ fluorescence probes, ${ }^{[7]}$ semiconductors ${ }^{[8]}$ or solar cell dyes. ${ }^{[9]}$

Organic nanomaterials are usually prepared by bottom-up approaches that involve the self-assembly of small organic molecules in a surrounding media driven by intermolecular interactions, namely, hydrogen bonding, $\pi-\pi$ stacking and electrostatic and hydrophilic-hydrophobic interactions, and are able to exhibit different morphologies with low dimensionality such as nanoparticles, nanofibers, nanorods, nanotubes and others. Size and shape of the nanomaterials strongly affect their properties, ${ }^{[10-12]}$ therefore, there is a great interest in controlling their morphology. When dealing with fluorescent organic nanomaterials, avoiding the decrease in emission efficiency that many $\pi$-conjugated molecules undergo due to aggregation (or concentration) quenching is a difficult challenge. Indeed, efforts are being focused on the study of molecules that show the opposite effect; that is, molecules that barely emit in liquid solution, but are highly luminescent in the solid state. ${ }^{[13,14]}$ Methods to obtain fluorescent organic nanostructures ${ }^{[15,16]}$ have been achieved from molecules that show the abnormal aggregation-induced enhancement of emission (AIEE) ${ }^{[17]}$ or aggregationinduced emission (AIE) phenomena. ${ }^{[18-20]}$ Recently, the term solid-state luminescence enhancement (SLE) ${ }^{[21]}$ has been proposed to subsume more precisely both the AIEE and AIE terms. 


\section{WILEY-VCH}

Fluorescent compounds based on the star-shaped 2,4,6-tris(1,2,3-triazol-4-yl)-1,3,5triazine (TTT) moiety have been widely studied in our group. They are readily prepared by a copper-catalyzed alkyne-azide coupling (CuAAC) click reaction and display promising properties in the field of functional materials due to their liquid crystal, fluorescence and electronic properties. ${ }^{[22,23]}$ The formation of well-organized columnar mesophases is highly favored when the TTT moiety is adequately functionalized at the periphery. ${ }^{[24]}$ The TTT moiety can indeed be used as a core for Hbonded supramolecular liquid crystals, ${ }^{[25]}$ leading to a modular approach to columnar mesophases that makes possible the design of ambipolar charge-transporting materials. ${ }^{[26]}$ Some TTT and other similar triazine derivatives have shown attractive behaviors not only in the bulk, but also in solution, showing to be able to selfaggregate in different solvents. ${ }^{[25,27]}$ This prompted us to investigate the self-assembly of TTT-based derivatives in the liquid phase, how to control the morphology of the aggregates and the consequences that the formation of the aggregates has on their optical properties. The TTT derivatives T3C $_{4}$ and TB3C $_{4}$ (Scheme 1), with aromatic triazine, triazole and phenyl rings, were foreseen as self-assembling moieties that can aggregate through intermolecular $\pi-\pi$ interactions in the appropriate solvent.

We describe herein the preparation of nanostructures with different morphologies and properties of the star-shaped $\mathbf{T 3 C}_{4}$ and $\mathbf{T B}_{4} \mathbf{C}_{4}$ TTT derivatives with terminal butoxy chains, which behave differently in their self-assembly and their fluorescent properties. The aggregates were prepared by employing a reprecipitation method using two solvent/non-solvent pairs at different ratios, giving rise to various remarkable morphologies with a significant impact on the optical behavior. Quantum-chemical calculations were also performed to gain insight into the aggregation and optical properties. 


\section{WILEY-VCH}

\section{Results and Discussion}

\subsection{Synthesis}

Compounds $\mathbf{T}_{3} \mathbf{C}_{4}$ and $\mathbf{T B}_{3} \mathbf{C}_{4}$ were both prepared following a previously described "one-pot" procedure. ${ }^{[22]}$ This consists on a CuAAC click reaction that uses as reagents 2,4,6tris[(trimethylsilyl)ethynyl]-1,3,5-triazine and the corresponding azides, 1-azido-4butoxybenzene or 4-azidophenyl 4-butoxybenzoate (1), occurring in situ the triply removal of the trimethylsilyl groups from the alkyne molecule and the coupling with the azides (Scheme 1). The synthesis of $\mathbf{T 3 C}_{4}$ was previously reported, ${ }^{[25]}$ whereas $\mathbf{T B}^{\left[\mathbf{C}_{4}\right.}$ is a novel compound that has been synthesized by adapting a procedure described in a previous work. ${ }^{[23]}$ In the latter case, the azide intermediate $\mathbf{1}$ was obtained through a Steglich coupling between 4azidophenol and 4-butoxybenzoic acid. In turn, 4-azidophenol was prepared by diazotization of 4-aminophenol and subsequent reaction with sodium azide (See the Supporting Information). ${ }^{[28]}$ Thanks to the versatility of the synthetic procedure, the two TTT derivatives incorporate two different structures linked to the triazole ring, $\mathbf{T 3 C}_{4}$ with three $p$ butoxyphenyl groups attached to the TTT core, and TB3C $_{4}$ with three $p$ butoxybenzoyloxyphenyl groups.
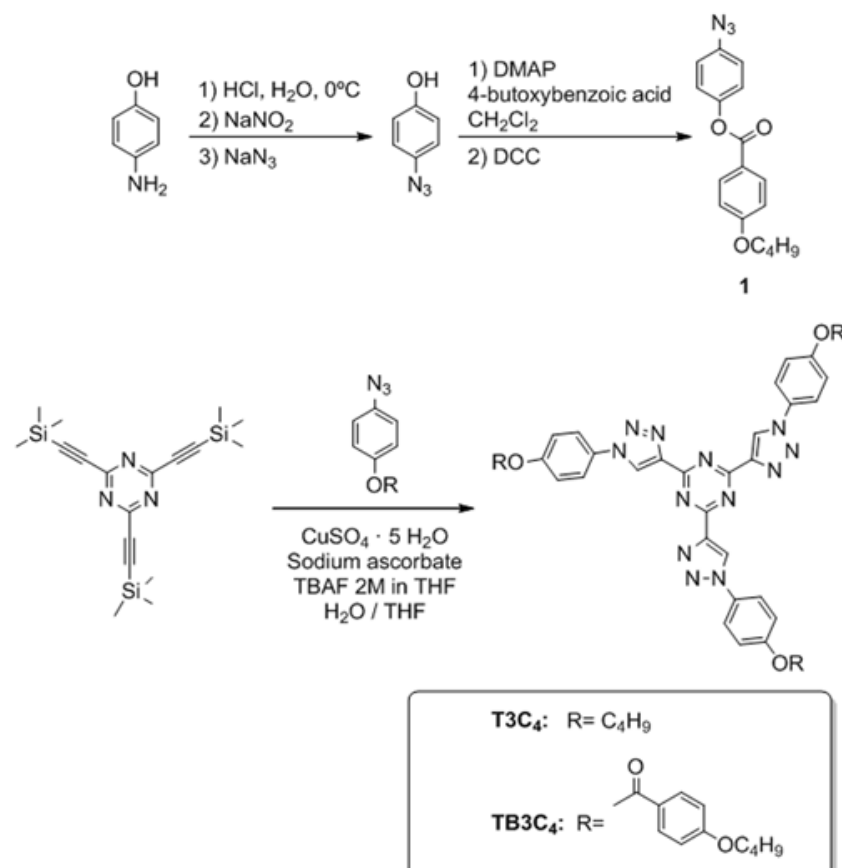


\section{WILEY-VCH}

Scheme 1. Synthesis of T3C $_{4}$ and TB3C $_{4}$.

\subsection{Computational insight into the self-assembly of $\mathrm{T}_{3} \mathrm{C}_{4}$ and $\mathrm{TB}_{3} \mathrm{C}_{4}$}

To investigate the relationship between the molecular structure of $\mathbf{T 3 C}_{4}$ and TB3C4 $_{4}$ and their self-assembly tendencies, supramolecular aggregates of both compounds were built and calculated at different quantum-chemical levels. The molecular geometries of the $\mathbf{T 3 C}_{4}$ and $\mathbf{T B}_{3} \mathbf{C}_{4}$ monomers were first optimized within $C_{3}$-symmetry constrains at the B3LYP-D3/6-31G** level. The minimum-energy structures optimized for both $\mathbf{T 3 C}_{4}$ and $\mathbf{T B}_{3} \mathbf{C}_{4}$ exhibit mostly planar conjugated cores, formed by the triazine and the triazole rings (the TTT core), with dihedral angles $\mathrm{D}_{1}$ of around $1^{\circ}$ (Figure 1). The butoxyphenyl peripheral groups of $\mathbf{T 3 C}_{4}$ are notably twisted with respect to the triazole rings with dihedral angles $\mathrm{D}_{2}$ of $29^{\circ}$. For $\mathbf{T B}_{3} \mathbf{C}_{4}$, the inner benzene rings are twisted by around $26^{\circ}$ with respect to the triazoles (dihedral angle $\mathrm{D}_{2}$ ) similarly to $\mathbf{T 3 C}_{4}$, and the terminal benzoyloxy groups are additionally rotated with a dihedral angle $\mathrm{D}_{3}$ of around $44^{\circ}$ (Figure 1). The rotation between the inner benzene and the benzoyloxy unit comes from the single character of the carbon-oxygen bond between both moieties. This rotation plays an important role in determining the optical properties of $\mathbf{T B}_{3} \mathbf{C}_{4}$ aggregates (vide infra).
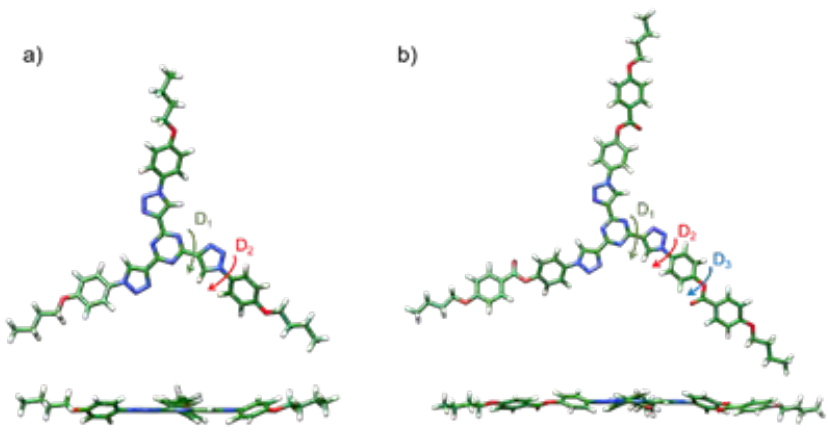

Figure 1. Top and side views of the B3LYP-D3/6-31G**-optimized structures calculated for $\mathbf{T 3 C}_{4}$ (a) and $\mathbf{T B 3 C}_{4}$ (b). Representative dihedral angles $\left(\mathrm{D}_{1}, \mathrm{D}_{2}\right.$ and $\left.\mathrm{D}_{3}\right)$ are highlighted. 


\section{WILEY-VCH}

Assisted by the structural information extracted from $\pi$-stacked $C_{3}$-trimer models of T3C $_{4}$ and TB3C $_{4}$ computed at the B3LYP-D3/6-31G** level (see the Supporting Information for further details), larger supramolecular aggregates (decamers) of T3C $_{4}$ and TB3C $_{4}$ were constructed and optimized (Figure 2) at the tightbinding density functional theory (DFTB3) level coupled to the Grimme's -D3 dispersion correction (DFTB3-D3). Two different structures were predicted for the T3C4 decamer at DFTB3-D3. The first optimized structure corresponds to a helicallike aggregate, in which adjacent molecules are disposed in a parallel fashion along the stacking axis and are rotated by angles in the $4-15^{\circ}$ range (Figure $2 \mathrm{a}$ ). The molecular units are slightly displaced in a zigzag manner with short $\pi-\pi$ contacts around $3.4-3.6$ A. In contrast, the second optimized structure of the $\mathbf{T 3 C}_{4}$ decamer shows an ellipsoidlike structure with significant folding of almost all the molecules forming the aggregate (Figure 2b). Despite its highly distorted structure, this second decamer presents some reminiscences of the helical-like structure obtained for the first decamer and for the DFT-optimized $\mathbf{T 3 C}_{4}$ trimer (Figure $2 \mathrm{a}$ and Figure S3a). The dihedral angles $\left(\mathrm{D}_{1}\right.$ and $\mathrm{D}_{2}$ ) previously used to characterize the molecular structure (Figure 1) exhibit values within a wide range due to the significant conformational disorder. Despite the disordered aspect of the aggregate, the structure predicted for the decamer still allows for favorable $\pi-\pi$ interactions between the triazine, triazole and benzene rings with intermolecular contacts in the 3.2-4.5 $\AA$ range (Figure 2c). In fact, this distorted ellipsoid-like decamer is found to be more stable by $36.6 \mathrm{kcal} / \mathrm{mol}$ than the helical-like $\mathrm{T}_{3} \mathrm{C}_{4}$ decamer.

For TB3C4, two stable decamer structures were also found. The first structure (Figure $2 \mathrm{~d}$ ) exhibits a helical-like aggregation mode mainly driven by $\pi-\pi$ interactions with short intermolecular contacts between adjacent triazine units $(\sim 3.4-3.6 \AA)$, 


\section{WILEY-VCH}

triazole rings $(\sim 3.6 \AA)$ and benzenes $(\sim 3.6 \AA)$. This decamer presents a relatively small conformational disorder with extended arms and values for the representative dihedral angles $D_{1}, D_{2}$ and $D_{3}$ in the same range as those obtained for the isolated molecule. The second structure also corresponds to a helical-like aggregate (Figure 2e), but in this case the TB3C $_{4}$ monomers are slightly displaced along the stack. This $\mathbf{T B}_{3} \mathbf{C}_{4}$ decamer resembles the trimer previously calculated at the B3LYP-D3/6-31G** level (Figure S3b) and is predicted to be $55.3 \mathrm{kcal} / \mathrm{mol}$ more stable than the decamer shown in Figure 2d. The slight shift of adjacent molecules along the stack, together with the high value of the twisting angle $D_{1}\left(\sim 22^{\circ}\right)$ found for one of the molecular arms, determines that two among the three arms become close and efficiently interact. In addition to the $\pi-\pi$ interactions between the aromatic rings in the $3.8-4.6 \AA$ range, complementary $\mathrm{CH}-\pi$ interactions are also present between the terminal alkyl chains and benzene rings.

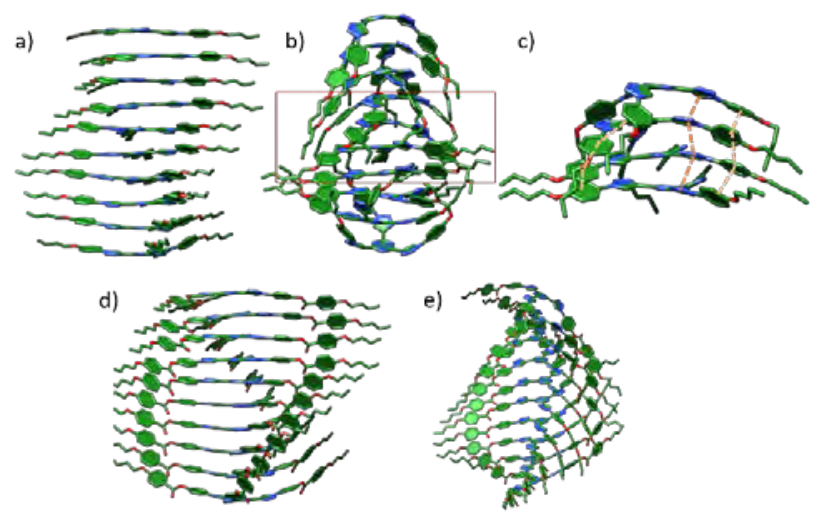

Figure 2. a-b) DFTB3-D3-optimized decamer structures calculated for T3C4.

(c) Magnification of the central four molecules of the optimized decamer of $\mathbf{T 3 C}_{4}$ shown in (b) to better visualize the intermolecular interactions. d-e) Helical-like DFTB3-D3-optimized decamers computed for TB3C4. Hydrogen atoms have been omitted for clarity. 


\section{WILEY-VCH}

DFTB3-D3 calculations therefore predict stable supramolecular aggregates for both T3C $_{4}$ and TB3C $_{4}$ governed by weak intermolecular $\pi-\pi$ and $\mathrm{CH}-\pi$ interactions between the aromatic rings of the TTT core and also between the peripheral butoxyphenyl (T3C4) and butoxybenzoyloxyphenyl (TB3C4) groups. Interestingly, calculations suggest that $\mathbf{T 3 C}_{4}$ and $\mathbf{T B 3 C}_{4}$ give rise, despite their similar molecular structure, to significantly different supramolecular assemblies. The more extended peripheral groups present in $\mathbf{T B 3 C}_{4}$ promote the formation of stable helical-type structures.

\subsection{Preparation of nanostructures and morphological characterization}

Encouraged by theoretical calculations that indicate energetically favored aggregation for both compounds with different structure, we explored the possibilities of controlling the formation of nanostructures.

It is well known that $\pi$-stacking causes the upfield shifting of the NMR signals due to the mutual shielding of the aromatic rings. ${ }^{[29-32]}$ Accordingly, a concentrationdependent ${ }^{1} \mathrm{H}-\mathrm{NMR}$ study in chloroform was carried out as a first step to explore this possibility. ${ }^{1} \mathrm{H}-\mathrm{NMR}$ spectra of $\mathbf{T 3 C}_{4}$ and $\mathbf{T B}_{3} \mathbf{C}_{4}$ in $\mathrm{CDCl}_{3}$ at different concentrations $\left(5 \times 10^{-4}, 2.5 \times 10^{-3}\right.$ and $\left.1 \times 10^{-1} \mathrm{M}\right)$ were recorded (Figure 3$)$. On increasing the concentration from $5 \times 10^{-4}$ to $2.5 \times 10^{-3} \mathrm{M}$, no changes were observed in the ${ }^{1} \mathrm{H}-\mathrm{NMR}$ signals, suggesting that this concentration is still too low for aggregation. Nevertheless, when the concentration was increased up to $1 \times 10^{-1} \mathrm{M}$, both the aromatic and the triazole protons of $\mathbf{T 3 C}_{4}$ and $\mathbf{T B}_{3} \mathbf{C}_{4}$ shifted upfield. This is in agreement with the short distances $(3.5-3.9 \AA)$ computed for the $\pi$-stacked trimer and decamer models (Figure S3 and Figure 2), and confirms that both compounds are able to self-assemble in chloroform above this concentration. The upfield shifting is more pronounced for TB3C $_{4}$, probably due to the presence of an additional aromatic benzoyloxy moiety, which makes more effective the interactions between the $\pi$-systems of adjacent 


\section{WILEY-VCH}

molecules in the aggregate. In the case of $\mathbf{T B}_{\mathbf{3}} \mathbf{C}_{4}$, a widening of proton signals was also observed resulting in a loss of resolution of the signals, additionally endorsing the aggregation. ${ }^{[29]}$ Based on the behavior observed in the ${ }^{1} \mathrm{H}-\mathrm{NMR}$ spectra, transmission electron microscopy (TEM) images were taken for samples of $\mathbf{T 3 C}_{4}$ and $\mathbf{T B}_{3} \mathbf{C}_{4}$ at $1 \times 10^{-1} \mathrm{M}$ in $\mathrm{CDCl}_{3}$ (Figure S4) revealing the formation of spherical nanoparticles in both cases, with sizes ranging from 200 to $1200 \mathrm{~nm}$ for $\mathbf{T 3 C}_{4}$ and from 500 to $1000 \mathrm{~nm}$ in the case of TB33 $_{4}$.
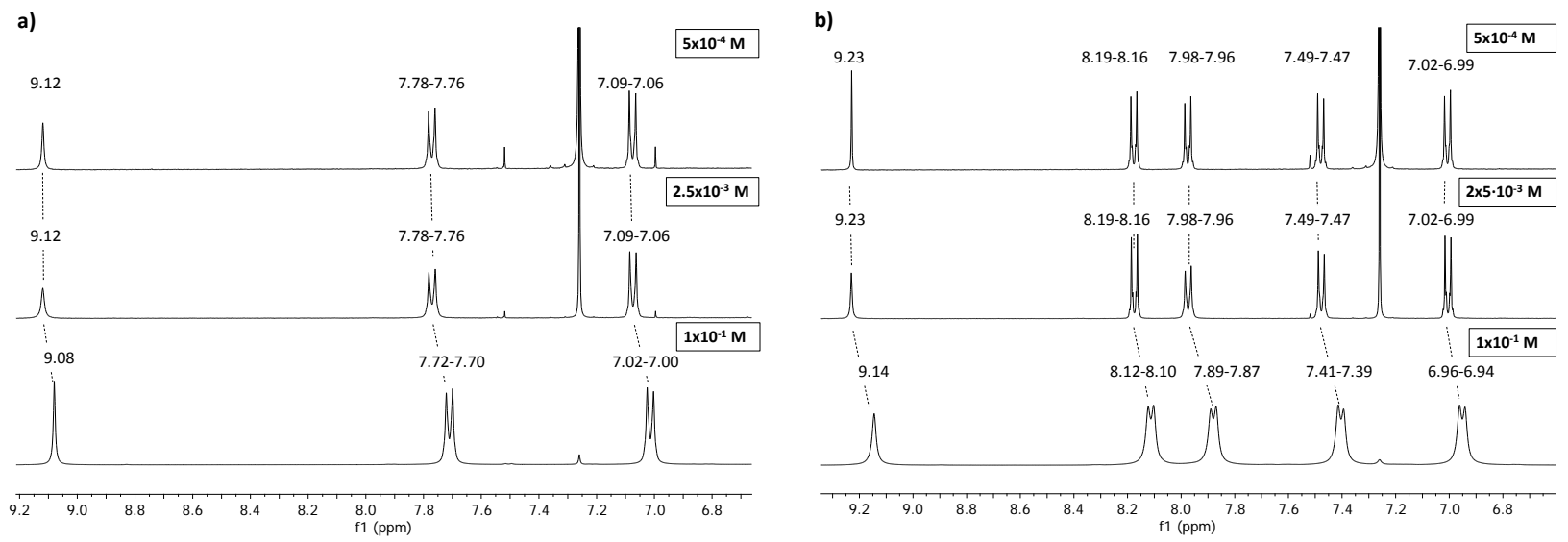

Figure 3. ${ }^{1} \mathrm{H}-\mathrm{NMR}$ spectra (aromatic region) of $\mathbf{T} 3 \mathbf{C}_{4}$ (a) and $\mathbf{T B} 3 \mathbf{C}_{4}$ (b) in $\mathrm{CDCl}_{3}$ at different concentrations.

To achieve fine control of the nanostructure size and morphology, we proposed the use of the reprecipitation method, which consists on a rapid mixing of a small volume of a concentrated solution of the organic compound with a miscible mixture of a good solvent and a non-solvent (See the Supporting Information). ${ }^{[1]}$ Thus, the molecules are quickly exposed to the presence of the non-solvent, causing the growth of the aggregates. This experimental procedure has been widely used for obtaining nanomaterials from organic molecules owing to its simplicity and versatility. ${ }^{[12,33]}$ Moreover, reprecipitation has been employed as a method to control the morphology of 


\section{WILEY-VCH}

the so-obtained nanostructures. ${ }^{[34]}$ In this work, two different solvent/non-solvent pairs were used, i.e. $\mathrm{CHCl}_{3} / \mathrm{MeOH}$ and $\mathrm{THF} / \mathrm{H}_{2} \mathrm{O}$.

T3C 4 formed aggregates in both $\mathrm{CHCl}_{3} / \mathrm{MeOH}$ and $\mathrm{THF} / \mathrm{H}_{2} \mathrm{O}$ mixtures. TEM and scanning electron microscopy (SEM) images from samples in $\mathrm{CHCl}_{3} / \mathrm{MeOH}$ revealed the formation of single-hollow spheres (Figure 4a-d). This morphology is quite rare for small organic molecules. ${ }^{[35,36]}$ The aggregates appeared to be more defined when the amount of $\mathrm{MeOH}$ was above $50 \%$. In addition, the size of the nanoparticles varied with the amount of non-solvent in the mixture: for samples with lower $\mathrm{MeOH}$ ratio (50\%), aggregates of 60-150 $\mathrm{nm}$ were observed, whereas when the non-solvent ratio was increased to $70 \%$, the particles grew up to $150-250 \mathrm{~nm}$. In a similar way, $\mathbf{T 3 C}_{4}$ aggregated in $\mathrm{THF} / \mathrm{H}_{2} \mathrm{O}$ mixtures at high water ratios. Above a $60 \%$ of water, well-defined single-hole hollow nanospheres with sizes ranging from 70 to $250 \mathrm{~nm}$ were formed as confirmed by SEM and TEM (Figure 4e-h). In TEM images, a round shell could be observed inside the nanosphere, indicating that they were hollow.

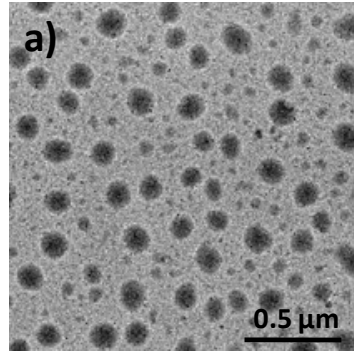

\section{b)}

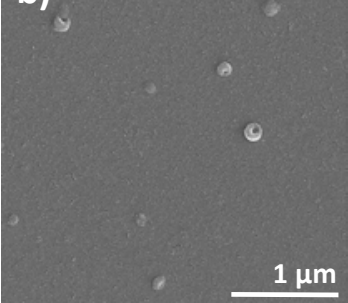

e)

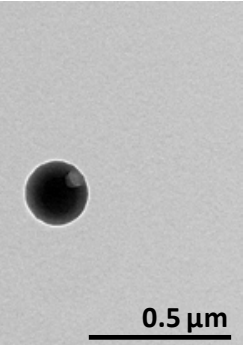

\section{f)}

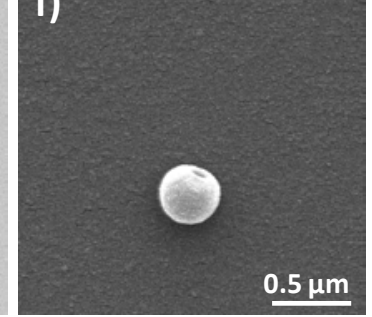

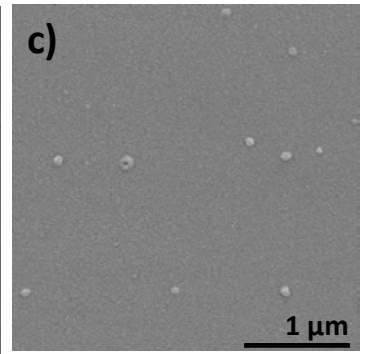

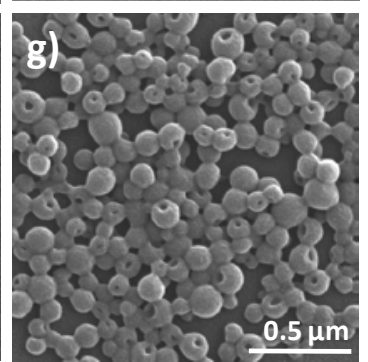

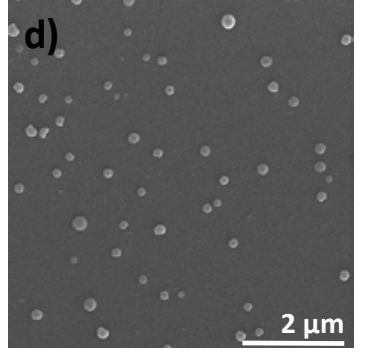

h)

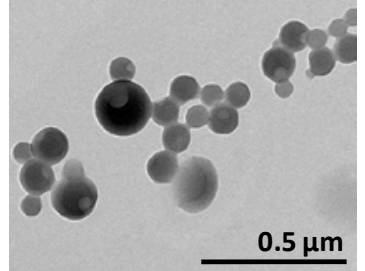

Figure 4. SEM (b, c, d, f and g) and TEM (a, e and h) images of T3C4 aggregates in $\mathrm{CHCl}_{3} / \mathrm{MeOH}$ 50/50 (a, b), $\mathrm{CHCl}_{3} / \mathrm{MeOH} 40 / 60$ (c), $\mathrm{CHCl}_{3} / \mathrm{MeOH}$ 30/70 (d), $\mathrm{THF} / \mathrm{H}_{2} \mathrm{O} 30 / 70(\mathrm{e}, \mathrm{f})$ and $\mathrm{THF} / \mathrm{H}_{2} \mathrm{O} 10 / 90(\mathrm{~g}, \mathrm{~h})$ mixtures. 


\section{WILEY-VCH}

For $\mathbf{T B}_{3} \mathrm{C}_{4}$ in $\mathrm{CHCl}_{3} / \mathrm{MeOH}$ mixtures, the amount of non-solvent in the solution strongly influenced the self-assembly process, giving rise to a variety of morphologies for the solvent mixtures containing $30 \% \mathrm{MeOH}$ or higher. For the solvent mixture containing $30 \%$ of $\mathrm{MeOH}$, spherical and toroid-like aggregates were detected, as well as other intermediate structures such as $\mathrm{Y}$-junctions, lariats or eights formed by two adjacent toroids (Figure 5a). These type of incomplete architectures, derived from the toroids, are commonly found when toroidal structures are formed. ${ }^{[37]}$ It is remarkable that hollow nanostructures such as one-hole spheres or toroids were obtained for both T3C $_{4}$ and TB3C4. These kind of nanostructures, unlike spheres or wires, are not so commonly detected because the range of solvent/non-solvent ratio in which they are stable is usually narrow. In the literature, toroids were normally described for amphiphilic polymers, dumbbell-shaped molecules or biological macromolecules (DNA or biopolymers), ${ }^{[37-41]}$ and recently they have been also reported for small organic molecules. ${ }^{[16,42]}$

When the amount of $\mathrm{MeOH}$ in the mixture was incremented, a drastic change in the morphology of the aggregates occurred, and the formation of twisted fibers of increasing size was observed. Firstly, short wires of 300-500 nm where observed when the amount of $\mathrm{MeOH}$ was $40 \%$ (Figure $5 \mathrm{~b}-\mathrm{c}$ ). Further increase to $50-90 \%$ of $\mathrm{MeOH}$ yielded longer fibers of several micrometer length (Figure 5d-f). These long fibers twisted forming screws, "phone cord-like" structures or helical nanotubes, scarcely reported in self-assembly systems from achiral molecules, ${ }^{[15]}$ as clearly revealed both SEM and TEM images. Occasionally, they closed on themselves giving rise to rings or entwined with other fibers in rope-like structures. 


\section{WILEY-VCH}
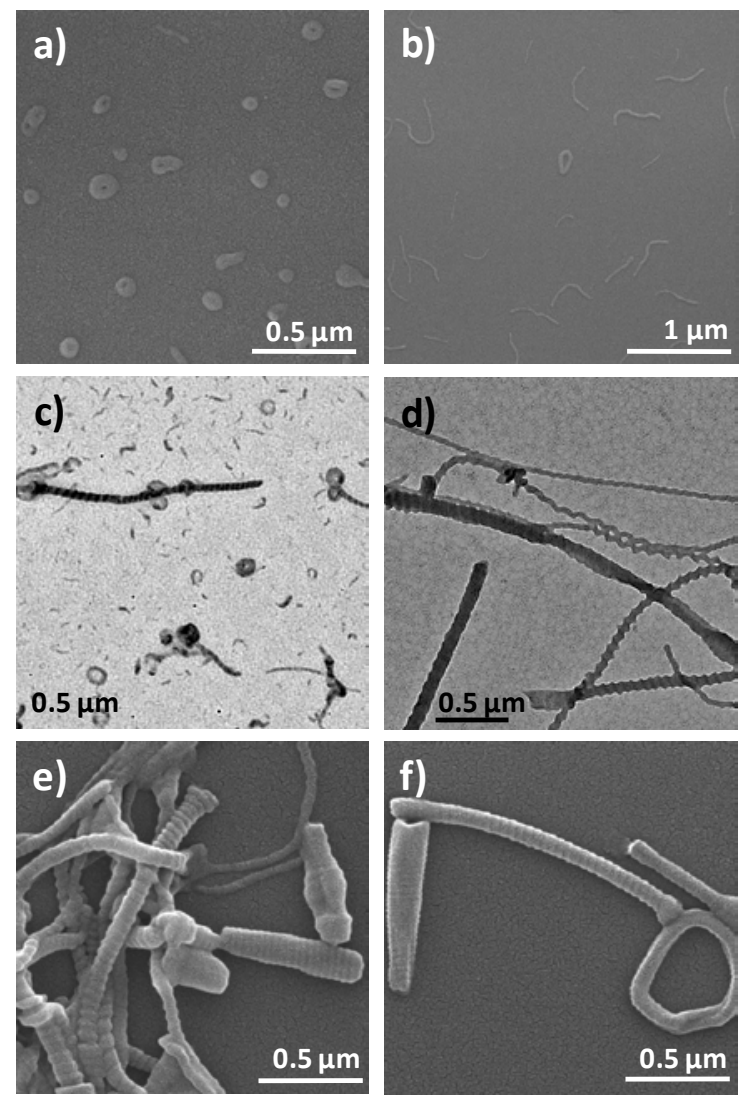

Figure 5. SEM (a, b, e and f) and TEM (c and d) images of TB3C 4 aggregates in $\mathrm{CHCl}_{3} / \mathrm{MeOH}$ 70/30 (a), $\mathrm{CHCl}_{3} / \mathrm{MeOH}$ 60/40 (b, c) and $\mathrm{CHCl}_{3} / \mathrm{MeOH}$ 20/80 (d, e, f) mixtures.

When the mixture of solvents was $\mathrm{THF} / \mathrm{H}_{2} \mathrm{O}$, aggregates were observed for water concentrations equal or above $60 \%$. As shown in TEM and SEM, the solvent mixture containing $60 \%$ of water allowed the formation of nanospheres of sizes ranging from 60 to $120 \mathrm{~nm}$ (Figure 6a-b). The increase of the amount of water in the mixture expanded the formation of the same particles (around $60 \mathrm{~nm}$ in size). Finally, when the concentration of water was augmented to $90 \%$, a large network of smaller spheres $(40-60 \mathrm{~nm})$ was observed (Figure 6c-d). Hence, in this case, the increase of the non-solvent in the mixture did not cause significant morphology changes compared to the $\mathrm{CHCl}_{3} / \mathrm{MeOH}$ solvent mixture. 


\section{WILEY-VCH}
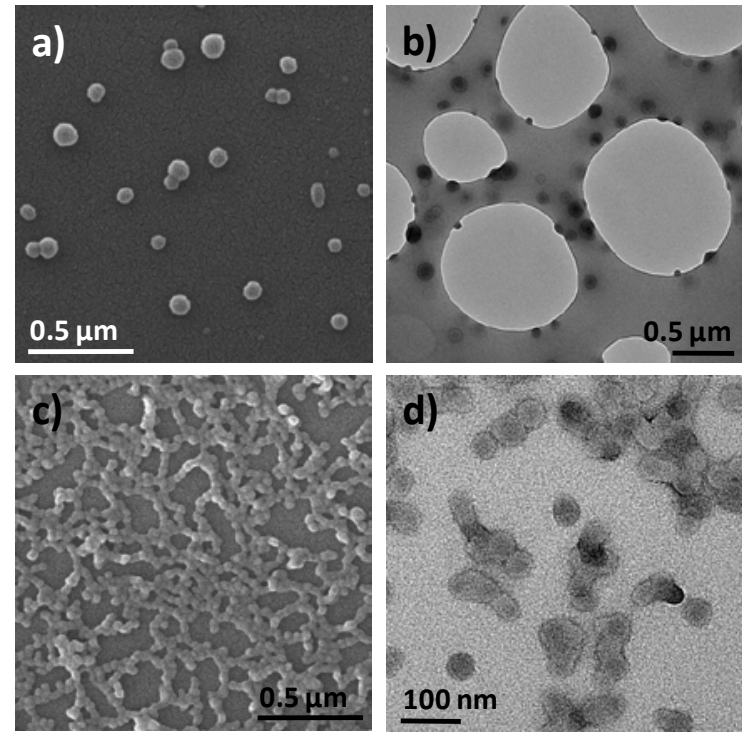

Figure 6. SEM (a and c) and TEM (b and d) images of TB3C 4 aggregates in $\mathrm{THF} / \mathrm{H}_{2} \mathrm{O}$ 40/60 (a, b) and THF/ $\mathrm{H}_{2} \mathrm{O}$ 10/90 (c, d) mixtures.

Experimental results can be tentatively related to B3LYP-D3 and DFTB3-D3 calculations that predicted different aggregation processes for $\mathbf{T}_{3} \mathbf{C}_{4}$ and $\mathbf{T B}_{3} \mathbf{C}_{4}$. T3 $\mathbf{C}_{4}$, owing to the favored folding of the molecular units (Figure 2b), may give rise to more rounded supramolecular assemblies, in line with the nanospheres observed in both solvent systems. In contrast, for $\mathbf{T B} \mathbf{C}_{4}$ more ordered and extended aggregates were calculated (Figure $2 \mathrm{~d}$ and e), which could make more easy the interaction with their surroundings, i.e. solvent molecules and vicinal aggregates. This may support the different aggregates experimentally observed for $\mathbf{T B 3 C}_{4}$ depending on the solvent system, forming wires, helical nanotubes or Y-junction nanoforms for the $\mathrm{CHCl}_{3} / \mathrm{MeOH}$ system and spherical objects in $\mathrm{THF} / \mathrm{H}_{2} \mathrm{O}$.

In light of the well-defined nanostructures observed by TEM and SEM, ${ }^{1} \mathrm{H}-\mathrm{NMR}$ experiments at different $\mathrm{CDCl}_{3} / \mathrm{CD}_{3} \mathrm{OD}$ and $\mathrm{THF}-\mathrm{d} 8 / \mathrm{D}_{2} \mathrm{O}$ ratios were performed for T3C $_{4}$ (Figure S5) and TB3C 4 (Figure 7) to bring additional data to understand the selfassembly process. Samples were prepared at a constant concentration of $1 \times 10^{-4} \mathrm{M}$ and tetrakis(trimethylsilyl)silane was employed as a chemical-shift standard. Figure 7 


\section{WILEY-VCH}

gathers the ${ }^{1} \mathrm{H}-\mathrm{NMR}$ spectra recorded for $\mathbf{T B}_{3} \mathbf{C}_{4}$ dissolved in each solvent mixture at four different solvent/non-solvent ratios. Interestingly, upon the addition of a small amount of the non-solvent, i.e. $20 \%$, a drastic intensity reduction is observed for all the signals indicating the decrease of molecularly dissolved TB3C $_{4}$ due to the selfassembly process. In addition, a significant down-field shift of the triazole proton (from 9.22 to $9.71 \mathrm{ppm}$ in $\mathrm{CDCl}_{3} / \mathrm{CD}_{3} \mathrm{OD}$ and from 9.62 to $9.91 \mathrm{ppm}$ in THF-d8/ $\mathrm{D}_{2} \mathrm{O}$ ) is observed, likely due to interaction with the protic non-solvent. Further increase of the relative amount of the non-solvent, $\mathrm{CD}_{3} \mathrm{OD}$ or $\mathrm{D}_{2} \mathrm{O}$, provokes the formation of assemblies that are NMR-silent, revealing the formation of solid-like nano-objects and confirming the presence of nanoaggregates in solution. This effect occurs at lower amount of the non-solvent for the mixture THF-d8/ $\mathrm{D}_{2} \mathrm{O}$.
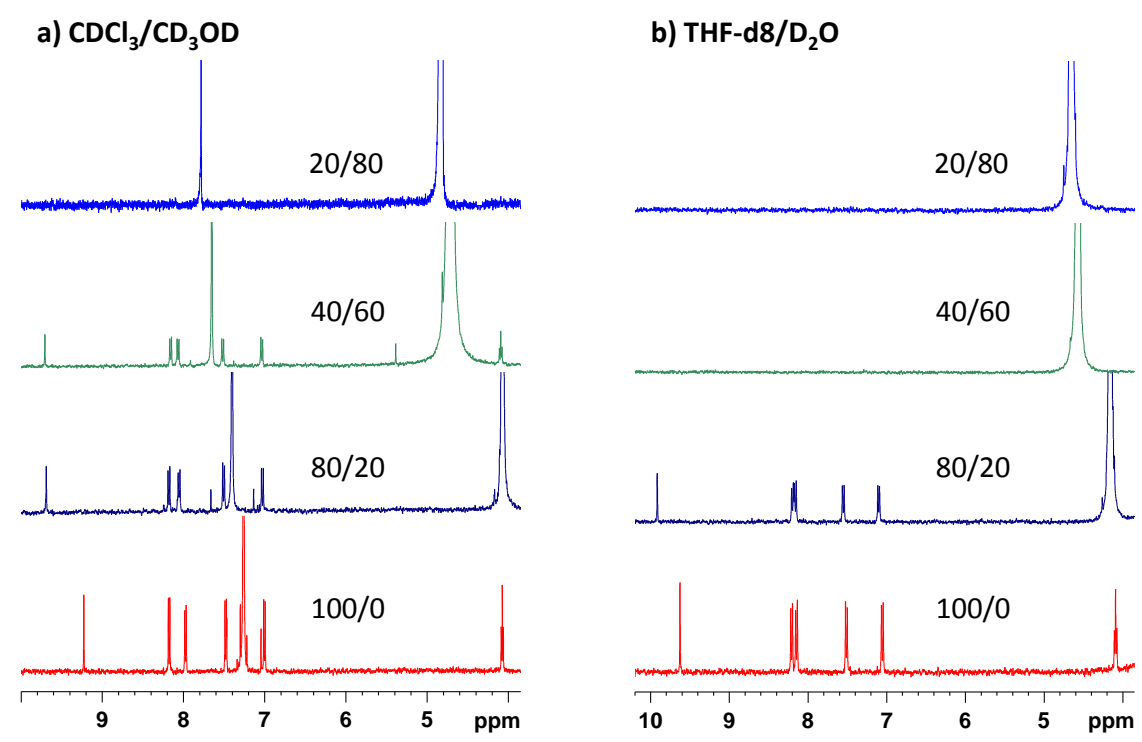

Figure 7. ${ }^{1} \mathrm{H}$ NMR spectra recorded for $\mathbf{T B}_{3} \mathbf{C}_{4}$ dissolved in solvent/non-solvent mixtures at different ratios and a concentration of $1 \times 10^{-4} \mathrm{M}$. a) $\mathrm{CDCl}_{3} / \mathrm{CD}_{3} \mathrm{OD}$ and b) THF-d8/D $2 \mathrm{O}$.

\subsection{Optical properties of $\mathrm{T}_{3} \mathrm{C}_{4}$ and $\mathrm{TB}_{3} \mathrm{C}_{4}$ aggregates}

Aggregation of optically active organic molecules has been shown to have a great influence in their photophysical features. Hence, the absorption and emission 


\section{WILEY-VCH}

spectra of $\mathbf{T 3 C}_{4}$ and $\mathbf{T B}_{3} \mathbf{C}_{4}$ were recorded in different solvent/non-solvent mixtures to investigate the possible changes caused in the optical properties by the formation of different nanostructures.

$\mathbf{T 3 C}_{4}$ is fluorescent in solution, exhibiting an emission band at $416 \mathrm{~nm}$ in $\mathrm{CHCl}_{3}$ with a quantum yield of 0.57 (Figure S6). However, the aggregation process caused by adding methanol produces a quenching of the emission. The same occurs for the $\mathrm{THF} / \mathrm{H}_{2} \mathrm{O}$ mixtures (Figure $\mathrm{S} 7$ and $\mathrm{S} 8$ ).

In contrast, $\mathbf{T B}_{3} \mathbf{C}_{4}$ shows a weak fluorescence band in $\mathrm{CHCl}_{3}\left(\lambda_{\mathrm{em}}=385 \mathrm{~nm}\right)$ or THF $\left(\lambda_{\mathrm{em}}=379 \mathrm{~nm}\right)$, which increases in the solvent mixtures containing aggregates (Figure S9). The plot in Figure 8a represents the dependence of the ratio $\mathrm{I} / \mathrm{I}_{0}(\mathrm{I}=$ fluorescence intensity measured at the different solvent/non-solvent mixtures; $\mathrm{I}_{0}=$ fluorescence intensity in pure $\mathrm{CHCl}_{3}$ solution) vs the percentage of $\mathrm{MeOH}$ in the solvent mixture. The fluorescence intensity (I) increased up to 7 times for the sample containing a $40 \%$ of $\mathrm{MeOH}$ in comparison with the compound dissolved in $\mathrm{CHCl}_{3}$ ( $\mathrm{I}_{0}$ ). Surprisingly, higher percentage of $\mathrm{MeOH}$ caused the emission to decrease again reaching a minimum for the sample containing $70 \% \mathrm{MeOH}$. The drop in emission coincides with a change in the morphology of the aggregates. Indeed, over $40 \%$ $\mathrm{MeOH}$, the spherical and toroidal aggregates disappeared, leading to the formation of short twisted fibers, which evolved to phone-cord like fibers and helical nanotubes of a few microns while increasing the amount of non-solvent. Thereby, aggregation of TB3C 4 in $\mathrm{CHCl}_{3} / \mathrm{MeOH}$ represents a new example of a compound with tuneable optical properties by means of control on the aggregation process.

More significant is the emission increase shown by $\mathbf{T B 3 C} 4$ when $\mathrm{THF} / \mathrm{H}_{2} \mathrm{O}$ mixtures are used. As depicted in Figure $8 \mathrm{~b}$, the fluorescence intensity of $\mathbf{T B}_{3} \mathbf{C}_{4}$ reached a maximum for the mixture containing an $80 \%$ of water. For this sample, the emission intensity (I) was more than 60 times higher than that of the solution in pure 


\section{WILEY-VCH}

THF $\left(\mathrm{I}_{0}\right)$, a value much greater than the obtained in the $\mathrm{CHCl}_{3} / \mathrm{MeOH}$ mixtures. An intense emission was also observed after removing the solvent for the dry sample (Figure S10). Anew, the influence of the morphology of the aggregates on the variation of the fluorescence was proven, since the mixture containing $90 \%$ of water again showed a slight decrease in the emission intensity, probably due to the formation of a less stable 3D-network of smaller spherical aggregates. $\mathbf{T B} \mathbf{C}_{4}$ therefore constitutes an interesting example of a compound that exhibits an enhancement of the emission by nanoparticle formation.

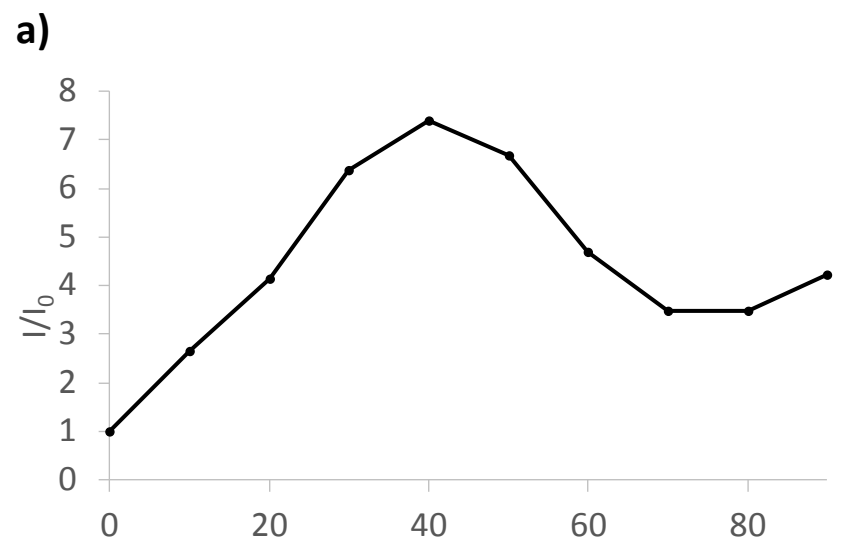

b)

$\mathrm{MeOH}(\%)$

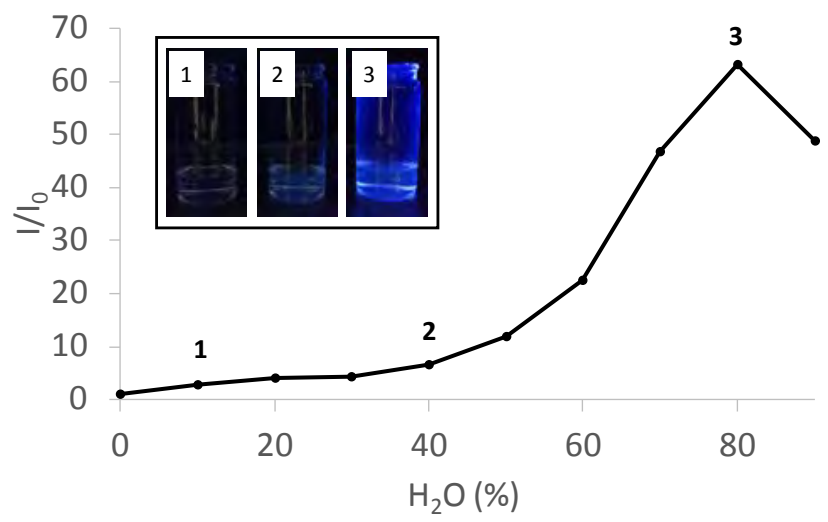

Figure 8 . Fluorescence intensity ratio $\left(\mathrm{I} / \mathrm{I}_{0}\right)$ as a function of the percentage of nonsolvent for TB3C 4 in $\mathrm{CHCl}_{3} / \mathrm{MeOH}$ (a) and $\mathrm{THF} / \mathrm{H}_{2} \mathrm{O}$ (b) mixtures. 


\section{WILEY-VCH}

It has been therefore observed that the optical properties of $\mathbf{T 3 C}_{4}$ and $\mathbf{T B}_{3} \mathbf{C}_{4}$ vary from those of the compounds molecularly dissolved when aggregates are obtained by using the reprecipitation method. Additionally, $\mathbf{T 3 C}_{4}$ and $\mathbf{T B}_{3} \mathbf{C}_{4}$ show different performances in terms of how aggregation affects the emission. One factor could be related to the different emitting properties of the non-aggregated $\mathbf{T}_{3} \mathbf{C}_{4}$ and $\mathbf{T B}_{3} \mathbf{C}_{4}$ compounds in solution; that is, $\mathbf{T 3 C}_{\mathbf{4}}$ is fluorescent whereas $\mathbf{T B} \mathbf{C}_{\mathbf{4}}$ hardly emits despite having the same emitting moiety: the TTT core. The structural difference is the presence of an extra benzoyloxy moiety, which adds flexibility to the molecule and could favor non-radiative deactivation channels leading to low emission efficiency. However, this deactivation pathway should be less favored when $\mathbf{T B}_{3} \mathbf{C}_{4}$ is in the aggregated state due to the rigid environment that could restrict the internal rotation, similar to what it has been postulated for other AIE molecules. ${ }^{[20]}$

To explore in more detail the intramolecular factors involved in the emission enhancement of $\mathbf{T B}_{3} \mathbf{C}_{4}$, relaxed torsion potentials of the most representative dihedral angles between the different aromatic rings were first calculated for the $\mathbf{T B}_{3} \mathbf{C}_{4}$ compound at the B3LYP-D3/6-31G** level. Figure 9 reveals that the rotation between the triazine and the triazole units (dihedral angle $\mathrm{D}_{1}$ ) is highly hindered, due to the disruption of the electronic conjugation between these rings, with an energy barrier of $9.1 \mathrm{kcal} / \mathrm{mol}$.

The rotation around the dihedral angle $\mathrm{D}_{2}$ (between the triazole and the benzene rings) is less impeded with barriers below $3.0 \mathrm{kcal} / \mathrm{mol}$. Importantly, the softest torsion potential is obtained for the dihedral angle $\mathrm{D}_{3}$ between the inner benzene and the benzoyloxy moiety with energy barriers of ca. $1.0 \mathrm{kcal} / \mathrm{mol}$. The benzoyloxy groups therefore rotate almost freely at room temperature $\left(\mathrm{k}_{\mathrm{B}} \mathrm{T} \sim 0.6 \mathrm{kcal} / \mathrm{mol}\right)$, and this rotation may represent a favorable mechanism for energy dissipation in the low-lying 


\section{WILEY-VCH}

electronic excited states. This would explain the fluorescence quenching of $\mathbf{T B} \mathbf{C}_{4}$ in solution, which presents a very low fluorescence quantum yield.

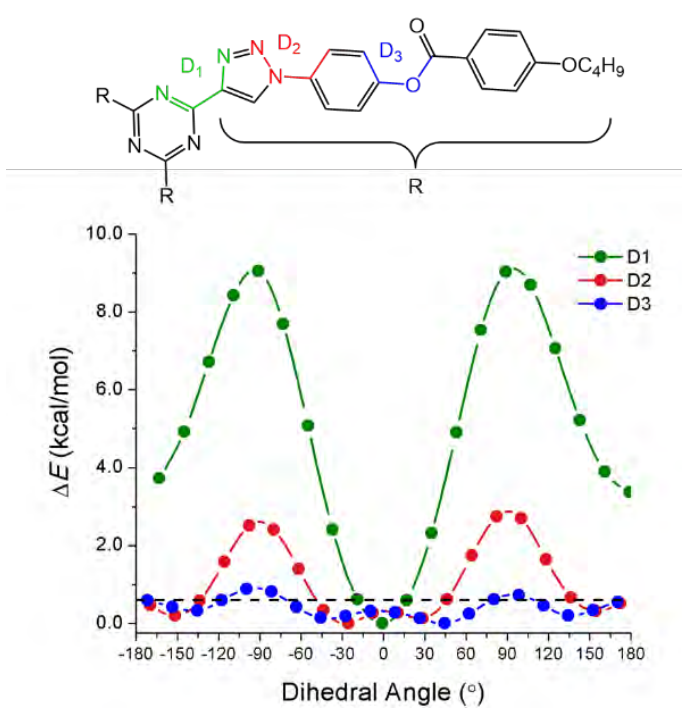

Figure 9. Relaxed torsion potentials calculated for selected dihedral angles $\left(D_{1}, D_{2}\right.$ and $\left.D_{3}\right)$ of TB3C $_{4}$ at the B3LYP-D3/6-31G** level. The horizontal dashed black line indicates $\mathrm{k}_{\mathrm{B}} \mathrm{T}$ energy at room temperature $(\sim 0.6 \mathrm{kcal} / \mathrm{mol})$.

In a second step, singlet excited states were computed from single-point timedependent DFT calculations (TDDFT) on the different relaxed structures obtained along the torsional $\mathrm{D}_{3}$ scan at B3LYP-D3/6-31G**. Figure 10 displays the excitation energies obtained for the first five singlet excited states $\left(\mathrm{S}_{1}\right.$ to $\left.\mathrm{S}_{5}\right)$ of $\mathbf{T B} \mathrm{C}_{4}$. The internal rotation involving the $\mathrm{D}_{3}$ angle gives rise to crossing between the low-lying excited states (particularly between the first three excited states) opening the door for dissipation pathways. For the molecular aggregates of $\mathbf{T B}_{3} \mathbf{C}_{4}$, the dihedral angle $\mathrm{D}_{3}$ is calculated to be in the $28-40^{\circ}$ range and the rotation of the benzoyloxy groups is hindered by the proximity of the adjacent molecules. In the $28-40^{\circ}$ interval of $\mathrm{D}_{3}$ values, the first singlet excited state for $\mathbf{T B} \mathbf{C}_{4}$ is a bright state with a high oscillator strength (Figure 10). This state would be therefore responsible for the enhanced 


\section{WILEY-VCH}

fluorescence emission observed experimentally due to the restriction of the torsion freedom. The restriction of intramolecular motions (in particular, hindered lowfrequency torsions) is the most common invoked mechanism to successfully explain the AIE phenomenon. ${ }^{[19]}$ This mechanism has been theoretically corroborated in molecular cluster models where the estimated non-radiative rate constants turned out to be smaller than those computed for the isolated systems. ${ }^{[43-45]}$

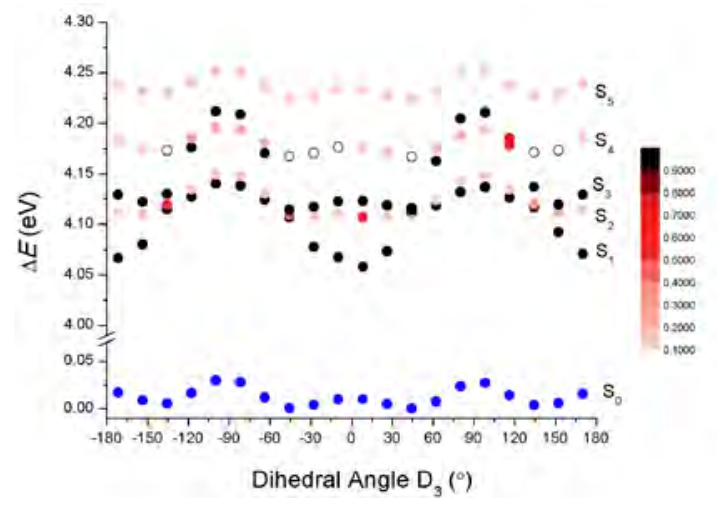

Figure 10. B3LYP-D3/6-31G** excitation energies calculated for the five lowest-lying singlet excited states $\left(\mathrm{S}_{\mathrm{n}}\right)$ of TB3C 4 as a function of the dihedral angle $\mathrm{D}_{3}$. The colour of the energy points represents the value of the oscillator strength as indicated on the scale on the

right. Ground state $\left(\mathrm{S}_{0}\right)$ energies in blue have been included for comparison purposes.

Our theoretical outcomes therefore highlight that, in the isolated $\mathbf{T B 3 C}_{4}$ compound, the torsion of the terminal benzoyloxy moieties has almost no energy cost at room temperature and, thus, is likely to be an efficient mechanism for fluorescence quenching. Singlet excited state calculations show that crossings between the low-lying excited states are expected through this rotation, giving rise to accessible non-radiative pathways. The restriction of the torsional motion in the molecular aggregates obstructs the energy-dissipating pathways and explains satisfactorily why $\mathbf{T B}_{\mathbf{3}} \mathbf{4}$ exhibits enhanced emission in the molecular assemblies. 


\section{WILEY-VCH}

\section{Conclusions}

This work reports on the use of the straightforward reprecipitation method to precisely control the morphology during the preparation of nanostructures from two non-amphiphilic star-shape 2,4,6-tris(1,2,3-triazol-4-yl)-1,3,5-triazines $\quad\left(\mathbf{T 3 C}_{4}\right.$ and TB3C4). Simple variations in some preparation parameters, such as the selection of the solvent/non-solvent pair or the relative amount of both solvents, lead to a variety of exciting architectures such as spherical particles, one-hole hollow spheres, toroids, twisted fibers or helical nanotubes.

The two derivatives allowed the study of the effect of the chemical structure on the aggregation and the optical properties. Compared to $\mathbf{T}_{3} \mathbf{C}_{4}$, the introduction of an additional benzoyloxy moiety with the ability to rotate in $\mathbf{T B}_{3} \mathbf{C}_{4}$ completely changed the emissive behavior. Whereas $\mathbf{T 3 C}_{4}$ acted as a conventional fluorescent molecule, in which aggregation caused the quenching of the emissive properties, $\mathbf{T B}_{3} \mathbf{C}_{4}$ represented an example of a molecule that exhibits enhancement of the emission upon aggregation. The control of the solvent/non-solvent mixture and thereby the morphology of the aggregate has been revealed as a tool for tuning the emission properties.

\section{Conflicts of interest}

There are no conflicts to declare.

\section{Acknowledgement}

This work was financially supported by the MINECO-FEDER (UE) funds (projects MAT2015-66208-C3-1, CTQ2015-71154-P, and Unidad de Excelencia María de Maeztu MDM-2015-0538), the Generalitat Valenciana (PROMETEO/2016/135 and SEJI/2018/035), the Gobierno de Aragón-FSE (UE) (E47_17R) and PI2 program from 


\section{WILEY-VCH}

ICMA-CSIC, M.C. and J.C. are grateful to the MINECO for their predoctoral contracts. J.A. is also thankful to the MINECO for a "JdC-Incorporación" Fellowship (IJCI-2015-26154). We would like to acknowledge the use of "Servicios CientíficoTécnicos" of CEQMA (UZ-CSIC), Servicio General de Apoyo a la Investigación SAI, Universidad de Zaragoza, and the Laboratory of Advanced Microscopies, LMAINA, Universidad de Zaragoza.

[1] Y. S. Zhao, H. Fu, A. Peng, Y. Ma, D. Xiao, J. Yao, Adv. Mater. 2008, 20, 2859.

[2] E. Busseron, Y. Ruff, E. Moulin, N. Giuseppone, Nanoscale 2013, 5, 7098.

[3] Y. Yan, Y. S. Zhao, Chem. Soc. Rev. 2014, 43, 4325.

[4] D. Yan, Chem. - A Eur. J. 2015, 21, 4880.

[5] D. Jiang, Z. Xue, Y. Li, H. Liu, W. Yang, J. Mater. Chem. C 2013, 1, 5694.

[6] S. Chen, Z. Qin, T. Liu, X. Wu, Y. Li, H. Liu, Y. Song, Y. Li, Phys. Chem. Chem. Phys. 2013, $15,12660$.

[7] F. Tang, C. Wang, J. Wang, X. Wang, L. Li, ACS Appl. Mater. Interfaces 2014, 6, 18337.

[8] M. Hasegawa, M. Iyoda, Chem. Soc. Rev. 2010, 39, 2420.

[9] S. Kundu, A. Patra, Chem. Rev. 2017, 117, 712.

[10] M. Abyan, D. De Caro, S. Fery-Forgues, Langmuir 2009, 25, 1651.

[11] S. P. Anthony, S. M. Draper, J. Phys. Chem. C 2010, 114, 11708.

[12] Y. Luo, Z. Xue, Y. Li, H. Liu, W. Yang, Y. Li, RSC Adv. 2015, 5, 100457.

[13] L. Maggini, D. Bonifazi, Chem. Soc. Rev. 2012, 41, 211.

[14] M. Martínez-Abadía, R. Giménez, M. B. Ros, Adv. Mater. 2017, 1704161.

[15] Y. Sang, P. Duan, M. Liu, Chem. Commun. 2018, 54, 4025.

[16] X. Jin, D. Yang, Y. Jiang, P. Duan, M. Liu, Chem. Commun. 2018, 54, 4513.

[17] B. K. An, S. K. Kwon, S. D. Jung, S. Y. Park, J. Am. Chem. Soc. 2002, 124, 14410. 


\section{WILEY-VCH}

[18] J. Luo, Z. Xie, J. W. Y. Lam, L. Cheng, B. Z. Tang, H. Chen, C. Qiu, H. S. Kwok, X. Zhan, Y. Liu, D. Zhu, Chem. Commun. 2001, 381, 1740.

[19] J. Mei, Y. Hong, J. W. Y. Lam, A. Qin, Y. Tang, B. Z. Tang, Adv. Mater. 2014, 26, 5429.

[20] J. Mei, N. L. C. Leung, R. T. K. Kwok, J. W. Y. Lam, B. Z. Tang, Chem. Rev. 2015, $115,11718$.

[21] J. Shi, L. E. Aguilar Suarez, S. J. Yoon, S. Varghese, C. Serpa, S. Y. Park, L. Lüer, D. Roca-Sanjuán, B. Milián-Medina, J. Gierschner, J. Chem. Phys. 2017, 121, 23166.

[22] E. Beltrán, J. L. Serrano, T. Sierra, R. Giménez, Org. Lett. 2010, 12, 1404.

[23] E. Beltrán, J. L. Serrano, T. Sierra, R. Giménez, J. Mater. Chem. 2012, 22, 7797.

[24] E. Beltrán, M. Garzoni, B. Feringán, A. Vancheri, J. Barberá, J. L. Serrano, G. M. Pavan, R. Giménez, T. Sierra, Chem. Commun. 2015, 51, 1811.

[25] B. Feringán, P. Romero, J. L. Serrano, R. Giménez, T. Sierra, Chem. - A Eur. J. 2015, $21,8859$.

[26] B. Feringán, P. Romero, J. L. Serrano, C. L. Folcia, J. Etxebarria, J. Ortega, R. Termine, A. Golemme, R. Giménez, T. Sierra, J. Am. Chem. Soc. 2016, 138, 12511.

[27] S. I. Kato, S. Jin, T. Kimura, N. Yoshikawa, D. Nara, K. Imamura, Y. Shiota, K. Yoshizawa, R. Katoono, T. Yamanobe, H. Uehara, Y. Nakamura, Org. Biomol. Chem. 2018, 16, 3584 .

[28] C. Courme, S. Gillon, N. Gresh, M. Vidal, C. Garbay, J. C. Florent, E. Bertounesque, Tetrahedron Lett. 2008, 49, 4542.

[29] G. Fernández, E. M. Pérez, L. Sánchez, N. Martín, Angew. Chemie - Int. Ed. 2008, 47, 1094.

[30] A. Pérez, D. De Saá, A. Ballesteros, J. L. Serrano, T. Sierra, P. Romero, Chem. - A Eur. J. 2013, 19, 10271.

[31] C. Zhang, C. Yu, H. Long, R. J. Denman, Y. Jin, W. Zhang, Chem. - A Eur. J. 2015, 21, 


\section{WILEY-VCH}

16935.

[32] Y. Takaki, R. Ozawa, T. Kajitani, T. Fukushima, M. Mitsui, K. Kobayashi, Chem. - A Eur. J. 2016, 22, 16760.

[33] M. Yang, P. Xing, M. Ma, Y. Zhang, Y. Wang, A. Hao, Soft Matter 2016, 12, 6038.

[34] K. Kazlauskas, G. Kreiza, E. Arbačiauskienė, A. Bieliauskas, V. Getautis, A. Šačkus, S. Juršènas, J. Phys. Chem. C 2014, 118, 25261.

[35] D. M. Li, Y. S. Zheng, J. Org. Chem. 2011, 76, 1100.

[36] Q. Li, H. Ma, Y. Jia, J. Li, B. Zhu, Chem. Commun. 2015, 51, 7219.

[37] D. J. Pochan, Z. Chen, H. Cui, K. Hales, K. Qi, K. L. Wooley, Science (80-. ). 2004, $306,94$.

[38] Z. Chen, H. Cui, K. Hales, Z. Li, K. Qi, D. J. Pochan, K. L. Wooley, J. Am. Chem. Soc. 2005, 127, 8592 .

[39] J. K. Kim, E. Lee, Z. Huang, M. Lee, J. Am. Chem. Soc. 2006, 128, 14022.

[40] E. Lee, Y. H. Jeong, J. K. Kim, M. Lee, Macromolecules 2007, 40, 8355.

[41] Y. B. Lim, E. Lee, M. Lee, Macromol. Rapid Commun. 2011, 32, 191.

[42] J. Cai, J. Liu, T. Wang, J. Wang, L. Jiang, J. Mater. Chem. C 2018, 6, 3849.

[43] Q. Peng, Y. Yi, Z. Shuai, J. Shao, J. Am. Chem. Soc. 2007, 129, 9333.

[44] Q. Wu, Q. Peng, Y. Niu, X. Gao, Z. Shuai, J. Phys. Chem. A 2012, 116, 3881.

[45] Q. Wu, C. Deng, Q. Peng, Y. Niu, Z. Shuai, J. Comput. Chem. 2012, 33, 1862. 


\section{WILEY-VCH}

\section{The table of contents entry}

Non-amphiphilic achiral star-shaped triazines self-assemble into hollow spheres, twisted fibres or phone cord-like helical nanotubes just by following a straightforward reprecipitation method. Control of the self-assembly morphologies and photoluminescent properties can be achieved, and aggregation induced emission is observed.

\section{Self-assembly}

Martín Castillo-Vallés, Eduardo Beltrán, Jesús Cerdá, Juan Aragó, Pilar Romero, José Luis Serrano, Enrique Orti, * Raquel Giménez* and Teresa Sierra*

\section{Self-assembly of clicked star-shaped triazines into functional nanostructures}

ToC figure

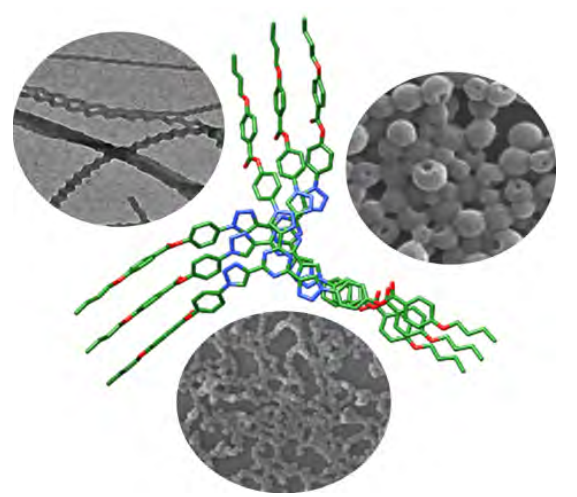

